\title{
Membrane structural changes support the involvement of mitochondria in the bile salt-induced apoptosis of rat hepatocytes
}

\author{
Susana SOLÄ, Maria A. BRITO*, Dora BRITES*, José J. G. MOURA† and \\ Cecilia M. P. RODRIGUES* \\ * Centro de Patogénese Molecular, Faculdade de Farmácia, University of Lisbon, Lisbon, Av. das Forças Armadas, \\ 1600-083 Lisbon, Portugal, and †Departamento de Química, Faculdade de Ciências e Tecnologia, Universidade Nova de Lisboa, \\ 2825 Monte de Caparica, Portugal
}

\section{A}

\begin{abstract}
The accumulation of toxic bile salts within the hepatocyte plays a key role in organ injury during liver disease. Deoxycholate (DC) and glycochenodeoxycholate (GCDC) induce apoptosis in vitro and in vivo, perhaps through direct perturbation of mitochondrial membrane structure and function. In contrast, ursodeoxycholate (UDC) and its taurine-conjugated form (TUDC) appear to be protective. We show here that hydrophobic bile salts induced apoptosis in cultured rat hepatocytes, without modulating the expression of pro-apoptotic Bax protein, and caused cytochrome $c$ release in isolated mitochondria. Co-incubation with UDC and TUDC prevented cell death and efflux of mitochondrial factors. Using spin-labelling techniques and EPR spectroscopy analysis of isolated rat liver mitochondria, we found significant structural changes at the membrane-water surface in mitochondria exposed to hydrophobic bile salts, including modified lipid polarity and fluidity, altered protein order and increased oxidative injury. UDC, TUDC and cyclosporin A almost completely abrogated DC- and GCDC-induced membrane perturbations. We conclude that the toxicity of hydrophobic bile salts to hepatocytes is mediated by cytochrome $c$ release, through a mechanism associated with marked direct effects on mitochondrial membrane lipid polarity and fluidity, protein order and redox status, without modulation of pro-apoptotic Bax expression. UDC and TUDC can directly suppress disruption of mitochondrial membrane structure, which may represent an important mechanism of hepatoprotection by these bile salts.
\end{abstract}

\section{INTRODUCTION}

The accumulation of hydrophobic bile salts within the hepatocyte appears to play a key role in organ injury during chronic liver diseases [1-3]. Although the loss of hepatocellular function has been attributed to necrosis, it is now clear that cell death induced by toxic bile salts such as deoxycholate (DC) or glycochenodeoxycholate (GCDC), both in vitro and in vivo, occurs quite often by apoptosis [4-8]. Several studies have further shown that cytotoxicity may be attributed to mitochondrial dysfunction. First, swollen mitochondria are common in bile duct-ligated rats and in humans with cholestasis [9], and mitochondria isolated from bile duct-ligated rats show impaired state III respiration [10]. Secondly, in vitro studies demonstrated that bile salts cause impaired state III respiration when added to permeabilized hepatocytes [11] or isolated mitochondria [12], while ATP depletion

Key words: Bax expression, EPR spectroscopy, hydrophobic bile salts, lipid polarity and fluidity, oxidative injury, protein order, tauroursodeoxycholate, ursodeoxycholate.

Abbreviations: DC, deoxycholate; DSA, doxyl stearic acid; GCDC, glycochenodeoxycholate; 4-maleimido-TEMPO, 4maleimido-2,2,6,6-tetramethylpiperidino-oxyl; RT-PCR, reverse transcription-PCR; TUDC, tauroursodeoxycholate; UDC, ursodeoxycholate.

Correspondence: Dr Cecília M. P. Rodrigues (e-mail cmprodrigues@ff.ul.pt). 
occurs during hepatocyte cell death [11]. Finally, mitochondrial perturbation due to toxic bile salts may be attributed to increased membrane permeability [7,13-15], whereas an elevation in the mitochondrial cardiolipin content has been shown to occur during cholestasis as an adaptive phenomenon to resist cell death caused by membrane permeabilization [16].

A common event in several models of cell death appears to be the opening of the permeability transition pore at the mitochondrial membrane, which may collapse the mitochondrial transmembrane potential, thus uncoupling the respiratory chain. In addition, several studies suggest that pro-apoptotic members of the Bcl-2 family, such as Bax, can also form pores in the mitochondrial outer membrane [17]. More recently, it was demonstrated that opening of the permeability transition pore signals the redistribution of cytosolic Bax protein to the mitochondria, where it forms clusters [18]. Although still disputed, cytochrome $c$ release from mitochondria is often regarded as being a consequence of mitochondrial perturbation. Toxic bile salts appear to induce both opening of the permeability transition pore $[7,13,14]$ and translocation of $\mathrm{Bax}[14,15]$, culminating in cytochrome $c$ release. Further, recent data have implicated Bid cleavage as the initiator of mitochondrial dysfunction in hepatocytes incubated with toxic bile salts [8]. Following entry into the cytosol, cytochrome $c$ promotes multiprotein complex formation, which induces proteolytic activation of cell death proteases known as caspases [19,20]. In addition, some consequences of increased mitochondrial permeability, such as changes in redox potential and primary activation of caspases, also favour membrane permeability, generating positive feedback loops [21].

In contrast with the biological toxic effects of hydrophobic bile salts, hydrophilic species such as ursodeoxycholate (UDC) and its amidated conjugates, tauroursodeoxycholate (TUDC) and glycoursodeoxycholate, ameliorate symptoms in patients with liver diseases (reviewed in [22]) and protect against the toxicity of hydrophobic bile salts (reviewed in [23]). Apoptotic cell death due to non-bile-salt-inducing agents is also partially prevented by UDC, supporting its unique role in regulating apoptosis through a mechanism involving mitochondrial function $[7,14,15]$. Indeed, UDC modulates the apoptotic threshold, in part, by inhibiting the opening of the mitochondrial permeability transition pore and by preventing translocation of Bax both in vitro and in vivo. Recent data have also provided evidence that UDC enters the mitochondria, suggesting that its antiapoptotic effect may be a consequence of a modulation of mitochondrial perturbation [24].

Based on these previous observations, we tested the hypothesis that hydrophobic bile salts act directly on mitochondrial membranes to induce permeabilization and cytochrome $c$ release. We investigated Bax expression in rat hepatocytes undergoing apoptosis induced by hydrophobic bile salts. In addition, we used EPR spectroscopy as a sensitive technique to examine changes in the lipid and protein structure of mitochondrial membranes associated with the release of intermembrane proteins, and to evaluate subsequent oxidative injury during exposure of isolated rat liver mitochondria to bile salts. This novel approach uses spin labels to obtain information about the polarity, fluidity and oxidation of the various domains of biomembranes, leading to accurate measurements of membrane integrity. The results demonstrate that hydrophobic bile salts induce apoptosis in cultured rat hepatocytes, without modulating proapoptotic Bax expression, and cause cytochrome $c$ release in isolated mitochondria. Moreover, the interaction of hydrophobic bile salts with the mitochondrial membrane resulted in disrupted lipid polarity and fluidity, increased protein order, and oxidative injury. Cytochrome $c$ release appears to also favour membrane permeability, perhaps generating vicious cycles. UDC, TUDC and cyclosporin A almost completely abrogated DC- and GCDC-induced perturbations of mitochondrial membrane structure. Thus the cytotoxicity of hydrophobic bile salts may be mediated, at least in part, by their direct effect of physically disturbing the mitochondrial membrane.

\section{MATERIALS AND METHODS}

\section{Isolation and culture of primary rat hepatocytes}

Cells were isolated from male Sprague-Dawley rats (200-250 g) by collagenase perfusion as described previously [25]. Briefly, rats were anaesthesized with phenobarbitol and their livers were perfused with $0.05 \%$ collagenase. Hepatocyte suspensions were obtained by passing digested livers through $125 \mu \mathrm{m}$-pore-size gauze and washing the cells in modified Eagle's medium (Atlanta Biologicals, Inc., Norcross, GA, U.S.A.). Cell viability was determined by Trypan Blue exclusion, and was typically $85-90 \%$. After isolation, hepatocytes were resuspended in William's E medium (Life Technologies, Inc., Grand Island, NY, U.S.A.) supplemented with $26 \mathrm{mM}$ sodium bicarbonate, $23 \mathrm{mM}$ Hepes, 0.01 unit/ $\mathrm{ml}$ insulin, $2 \mathrm{mM}$ L-glutamine, $10 \mathrm{nM}$ dexamethasone, $5.5 \mathrm{mM}$ glucose, 100 units $/ \mathrm{ml}$ penicillin and 100 units $/ \mathrm{ml}$ streptomycin, and then plated on Primaria ${ }^{\mathrm{TM}}$ tissue culture dishes (Becton Dickinson Labware, Lincoln Park, NJ, U.S.A.) at $1.0 \times 10^{6}$ cells $/ \mathrm{ml}$. Cells were maintained at $37^{\circ} \mathrm{C}$ in a humidified atmosphere of $5 \% \mathrm{CO}_{2}$ for $3 \mathrm{~h}$. Plates were then washed with medium to remove dead cells, and incubated in William's E medium containing $10 \%(\mathrm{v} / \mathrm{v})$ heat-inactivated foetal bovine serum (Atlanta Biologicals). All animal procedures were in accordance with the NIH Guide for the Care and Use of Laboratory Animals, and were approved by our Institutional Animal Care and Use Committee. 


\section{Induction of apoptosis and morphological analysis}

Freshly isolated hepatocytes were cultured as described above and then incubated with William's E medium supplemented with either $50 \mu \mathrm{M} \mathrm{DC}$ (prepared in water) or $50 \mu \mathrm{M}$ GCDC (prepared in DMSO) for $4 \mathrm{~h}$, with or without $50 \mu \mathrm{M}$ UDC or $50 \mu \mathrm{M}$ TUDC (Sigma Chemical Co., St. Louis, MO, U.S.A.). In co-incubation studies, cells were pretreated for $1 \mathrm{~h}$ with hydrophilic bile salts, and these agents were left in the medium when hydrophobic bile salts were added. Appropriate controls treated with water or DMSO $(<1 \%)$ vehicle only were also included. The medium was gently removed at the end of each incubation period and scored for non-viable cells by Trypan Blue dye exclusion, while attached cells were fixed for morphological evaluation of apoptotic changes. In brief, cells were fixed with $4 \%$ formaldehyde in PBS, pH 7.4, for $10 \mathrm{~min}$ at room temperature, incubated with Hoechst dye 33258 (Sigma Chemical Co.) at $5 \mathrm{mg} / \mathrm{ml}$ in PBS for $5 \mathrm{~min}$, washed with PBS and mounted using PBS/glycerol $(3: 1, \mathrm{v} / \mathrm{v})$. Fluorescence was visualized using an Axioskop ${ }^{\circledR}$ fluorescence microscope (Carl Zeiss G.m.b.H., Jena, Germany). Fluorescent nuclei were scored by different workers and categorized according to the condensation and staining characteristics of chromatin. Normal nuclei showed non-condensed chromatin dispersed over the entire nucleus. Apoptotic nuclei were identified by condensed chromatin, contiguous to the nuclear membrane, as well as by nuclear fragmentation of condensed chromatin. Three random microscopic fields per sample of approx. 200 nuclei were counted, and mean numbers of apoptotic nuclei were expressed as a percentage of total nuclei.

\section{RNA isolation and reverse transcription-PCR (RT-PCR)}

Bile salt-induced changes in Bax expression in rat primary hepatocytes were determined by RT-PCR. Total RNA was extracted from cells exposed to bile salts as described above using TRIZOL ${ }^{\mathrm{TM}}$ reagent (Life Technologies Inc.). For RT-PCR, $5 \mu \mathrm{g}$ of total RNA was reverse-transcribed using oligo(dT) (IDT, Inc., Coralville, IA, U.S.A.) and SuperScript ${ }^{\mathrm{TM}}$ II reverse transcriptase (Life Technologies Inc.). Specific oligonucleotide primer pairs were incubated with a cDNA template for PCR amplification using the Expand High Fidelity PCR System from Roche Diagnostics (Mannheim, Germany). The following sequences were used as primers: Bax sense primer, 5'-TGGTTGCCCTTTTCTACTTTG-3'; Bax antisense primer, $5^{\prime}$-GAAGTAGGAAAGGAGGCCATC- ${ }^{\prime}$; $\beta$ actin sense primer, $5^{\prime}$-TGCCCATCTATGAGGGTTACG-3'; $\beta$-actin antisense primer, 5'-TAGAAGCATTTGCGGTGCACG-3'. The product of constitutively expressed $\beta$-actin mRNA served as a control.

\section{Isolation of rat liver mitochondria and detection of cytochrome $c$ release}

Low-calcium liver mitochondria were isolated from male Sprague-Dawley rats (200-250 g) as described previously $[13,14]$. Following isolation, mitochondria were kept on ice for up to $3 \mathrm{~h}$. Protein concentrations were determined using the Bio-Rad protein assay (Bio-Rad Laboratories, Hercules, CA, U.S.A.).

Isolated mitochondria $(1 \mathrm{mg} / \mathrm{ml})$ were incubated in respiration buffer $(10 \mathrm{mM}$ Hepes, $10 \mathrm{mM}$ succinate, $215 \mathrm{mM}$ mannitol, $71 \mathrm{mM}$ sucrose, $\mathrm{pH}$ 7.4), pretreated with Chelex-100 (Bio-Rad Laboratories), in the presence of 50-500 $\mu \mathrm{M} \mathrm{DC}$ or GCDC for $5 \mathrm{~min}$, with or without $500 \mu \mathrm{M}$ UDC, $500 \mu \mathrm{M}$ TUDC or $5 \mu \mathrm{M}$ cyclosporin $\mathrm{A}$ (Sigma Chemical Co.). In co-incubation studies, mitochondria were pretreated for $5 \mathrm{~min}$, and all agents were present in the respiration buffer throughout the experiment. Appropriate controls treated with water or DMSO $(<1 \%)$ vehicle only were also included. Mitochondrial suspensions were then spun down at $12000 \mathrm{~g}$ for $3 \mathrm{~min}$ at $4{ }^{\circ} \mathrm{C}$. For detection of cytochrome $c$ release, aliquots of the supernatant and pellet were subjected to SDS/PAGE. Proteins were separated on a $15 \%(\mathrm{w} / \mathrm{v})$ polyacrylamide gel, transferred on to nitrocellulose membranes, and the immunoblots were treated with $15 \% \mathrm{H}_{2} \mathrm{O}_{2}$ for $15 \mathrm{~min}$ at room temperature. Blots were then incubated sequentially with $5 \%(\mathrm{w} / \mathrm{v})$ milk blocking solution, with primary monoclonal antibody to cytochrome $c$ (PharMingen, San Diego, CA, U.S.A.) at a dilution of 1:5000 overnight at $4{ }^{\circ} \mathrm{C}$, and finally with secondary goat anti-mouse $\operatorname{IgG}$ antibody conjugated with horseradish peroxidase (Bio-Rad Laboratories) for $3 \mathrm{~h}$ at room temperature. Membranes were processed for cytochrome $c$ detection using the enhanced chemiluminescence light $\left(\mathrm{ECL}^{\mathbb{R}}\right)$ reagent from Amersham Life Science, Inc. (Arlington Heights, IL, U.S.A.).

\section{EPR spectroscopy and spin-labelling techniques}

Changes in mitochondrial membrane structure were assessed by EPR spectroscopy using paramagnetic reporter groups or spin labels. Characteristics of mitochondrial membrane polarity at various depths were examined by using three different stearic acid spin labels, 5-doxyl stearic acid (5-DSA), 7-DSA and 16-DSA (Sigma Chemical Co.), containing the nitroxide group at different positions along the hydrocarbon chain of the stearic acid molecule [26-28]. The paramagnetic centres of 5- and 16-DSA spin labels are, respectively, near the lipid-water interface and deep within the lipid bilayer, and incorporation of these spin probes into mitochondrial membranes results in distinctive spectra upon excitation with microwaves in a magnetic field. A suitable parameter of the environment of these spin labels, calculated from direct measurements of the parallel and 
perpendicular components of the hyperfine tensor of the spin label, is the isotropic splitting factor $a_{0}$ [27]. Changes in the parameter of the order of only $2 \%$ are considered, and have been tested, to be significant. Increased polarity is reflected by a high $a_{0}$ value. In addition, alterations in the membrane dynamic properties of 5- and 7-DSA spin labels, showing restricted motion in the membrane, were evaluated by measuring the outer half-width at halfheight of the low-field extreme $(\Delta l)$. The larger the $\Delta l$ value, the more motion and less order in the local microenvironment reported by the nitroxide group. This parameter is more sensitive than the classical parameter $S$, and is independent of changes in the polarity of the environment around the nitroxide, rendering corrections unnecessary [29,30]. For the 16-DSA spin label, which showed a higher degree of motional freedom, the ratio of the heights of the low-field and centre-field lines $\left(b_{+1} / b_{0}\right)$ was used as an empirical measurement of membrane lipid organization [31,32]. An increased ratio reflects a decrease in membrane organization. Moreover, we used the 4-maleimido-2,2,6,6-tetramethylpiperidino-oxyl (4maleimido-TEMPO) label, which binds to the thiol groups of proteins, and is therefore suitable for measuring changes in the mobility of protein reactive groups. This spin label is particularly useful for the measurement of changes in the structure of protein-rich membranes $[28,33,34]$. The ratio of the height of the midline to the height of the high-field line of the spectrum $\left(h_{0} / h_{-1}\right)$ gives the information about the freedom of motion of this spin label at its binding site, which in turn provides a parameter for the mobility of thiol groups within the membrane. A low freedom of motion is indicated by a high $h_{0} / h_{-1}$ ratio.

All spin labels were dissolved in chloroform, separated into $1 \mu \mathrm{g}$ aliquots, evaporated under nitrogen and stored under vacuum for at least $2 \mathrm{~h}$. The mitochondrial suspension containing 100-150 $\mu \mathrm{g}$ of protein was incubated in spin-label-coated tubes with gentle shaking for 90 min at $37^{\circ} \mathrm{C}(5-, 7$ - and $16-D S A)$, or for 1 min at $22^{\circ} \mathrm{C}$ (4-maleimido-TEMPO), yielding systems containing approx. $5 \mathrm{~mol}$ of spin label per $100 \mathrm{~mol}$ of membrane lipid ( $5 \mathrm{~mol} \%$ probe). Under these conditions, the incorporation of the spin label into the membrane was constant, while distortion of the spectra due to spin-spin interaction was not noted. In addition, levels of incorporation were controlled by double integration of the spectra, ensuring that results were independent of the spin-label concentration. Finally, incorporation of spin labels did not significantly damage liver mitochondria. Release of glutamate dehydrogenase and translocation of cytochrome $c$ in controls did not change appreciably during incubation with spin labels.

Mitochondrial membrane lipid peroxidation was also investigated using EPR spectroscopy and paramagnetic reporter groups incorporated into membranes [35-37]. In short, freshly isolated mitochondria $(5-10 \mu \mathrm{g}$ of protein) were incubated with two membrane-associated, oxidation-sensitive, paramagnetic nitroxyl stearate probes (1 $\mu$ g aliquots), i.e. 5- and 12-DSA, with gentle agitation for $20 \mathrm{~min}$ at $22{ }^{\circ} \mathrm{C}$. Increased production of reactive oxygen species results in loss of paramagnetism of the spin label and hence loss of signal amplitude, measured as the height of the centre-field line of the spectrum, providing that the shape remains the same.

Mitochondrial membranes thus labelled were exposed to $50-1000 \mu \mathrm{M} \mathrm{DC}$ or GCDC in Chelex-100-treated respiration buffer for $5 \mathrm{~min}$ at $22^{\circ} \mathrm{C}$, yielding a molar ratio of approx. $100 \mathrm{~mol}$ of bile salts $/ 100 \mathrm{~mol}$ of endogenous lipids in most experiments. In co-incubation studies, probed mitochondria were pretreated for $5 \mathrm{~min}$ with $500 \mu \mathrm{M}$ UDC, $500 \mu \mathrm{M}$ TUDC or $5 \mu \mathrm{M}$ cyclosporin $A$, and these agents were left in the respiration buffer when the hydrophobic bile salts were added. Appropriate controls treated with water or DMSO $(<1 \%)$ vehicle only were also included. The order of lipophilicity of the bile salts using the octanol/water partition coefficient is DC $(3 \alpha, 12 \alpha$-dihydroxy- $5 \beta$-cholanoic acid $)>$ chenodeoxycholate $(3 \alpha, 7 \alpha$-dihydroxy- $5 \beta$-cholanoic acid $)>$ UDC ( $3 \alpha, 7 \beta$-dihydroxy- $5 \beta$-cholanoic acid) for both the protonated and ionized unconjugated and glycineamidate bile acids [38].

At the end of the exposure period, the mitochondrial suspension was spun down at $12000 \mathrm{~g}$ for $3 \mathrm{~min}$, and the pellet was used for EPR spectroscopy analysis. In brief, mitochondrial pellets were resuspended in respiration buffer $(60 \mu \mathrm{l})$ and sucked into $100 \mu \mathrm{l}$ glass capillaries, which were sealed at both ends, and the capillaries were introduced into standard 4-mm quartz tubes containing silicone oil for thermal stability. All spectra were acquired at $9.8 \mathrm{GHz}$ (X-band) on a Bruker EMX EPR spectrometer (Bruker, Karlsruhe, Germany) using a rectangular cavity (model ER $4102 \mathrm{ST}$ ) and $100 \mathrm{kHz}$ field modulation frequency, $1.05 \mathrm{G}$ modulation amplitude and $20 \mathrm{~mW}$ microwave power, at $22^{\circ} \mathrm{C}$.

\section{Densitometry and statistical analysis}

Densitometry analysis was accomplished using a PC coupled to a PRIMAX ${ }^{\mathrm{TM}} 9600$ Profi VM6575 scanner (Primax International B.V., Utrecht, The Netherlands). Quantification of the autoradiograms used the ImageMaster 1D Elite densitometric analysis program (Amersham Pharmacia Biotech, Uppsala, Sweden). Fold and percentage changes in EPR spectroscopy parameters were always calculated based on the corresponding controls to minimize the ambiguity due to binding of molecules to both mitochondrial outer and inner membranes. All data are expressed as means \pm S.E.M. from at least four separate experiments. Differences between groups were compared using the unpaired two-tailed Student's $t$ test, performed on the basis of equal or unequal variance as appropriate, and $P$ values of $<0.05$ were considered statistically significant. 


\section{RESULTS}

\section{Is bile salt-induced apoptosis of hepatocytes associated with altered expression of pro- apoptotic Bax?}

In initial experiments, we confirmed that significant levels of apoptosis occurred in cultured primary rat hepatocytes after incubation with hydrophobic bile salts [7]. Incubation of cells with $50 \mu \mathrm{M}$ DC resulted in a significant increase in the number of apoptotic cells, from less than $1 \%$ in controls to approx. $5 \%$ and $12 \%$ at 4 and $6 \mathrm{~h}$ of incubation respectively $(P<0.01)$. Few hepatocytes were apoptotic after $4 \mathrm{~h}$ of exposure to $50 \mu \mathrm{M}$ GCDC, but almost $8 \%$ displayed nuclear fragmentation and condensed chromatin following incubation with $100 \mu \mathrm{M}$ GCDC $(P<0.05)$. Incubation with UDC or TUDC alone produced no significant apoptotic changes compared with controls. In addition, both of the hydrophilic bile salts almost completely protected against apoptosis induced by the hydrophobic species, reducing cell death by more than $80 \%(P<0.01)$.

We have shown previously that exposure of hepatocytes to DC resulted in translocation of Bax to the mitochondria, both in vitro and in vivo, while UDC modulated the apoptotic threshold, in part, by preventing relocation of the pro-apoptotic protein $[14,15]$. Here we investigated whether bile salt-induced apoptosis is related to changes in expression of Bax, and if hydrophilic bile salts mediate cell death by influencing gene transcription. Our results indicate that bile salts did not significantly modulate the expression of Bax, as assessed by RT-PCR in isolated hepatocytes. In fact, more hydrophobic molecules such as DC induced only a marginal $5 \%$ increase in Bax mRNA levels compared with controls, whereas UDC and TUDC resulted in increases of less than $2.5 \%$. Thus it appears that bile salts markedly influence the translocation of Bax from cytosol to mitochondria during apoptosis without modulating its gene expression.

\section{Do hydrophobic bile salts disturb lipid and protein structure in isolated liver mitochondria, and, if so, can this be prevented by hydrophilic bile salts?}

Although our results indicated that hydrophobic bile salts induce apoptosis in cultured rat hepatocytes, these bile salts did not significantly modulate the expression of pro-apoptotic Bax. In subsequent experiments, we characterized the direct disturbing effects of bile salts on mitochondria, perhaps leading to cell death. The interaction with the lipid domain of mitochondrial membranes was investigated using EPR spectroscopy and several stearic acid spin labels. Bile salt concentrations were selected based on the general belief that toxicity is

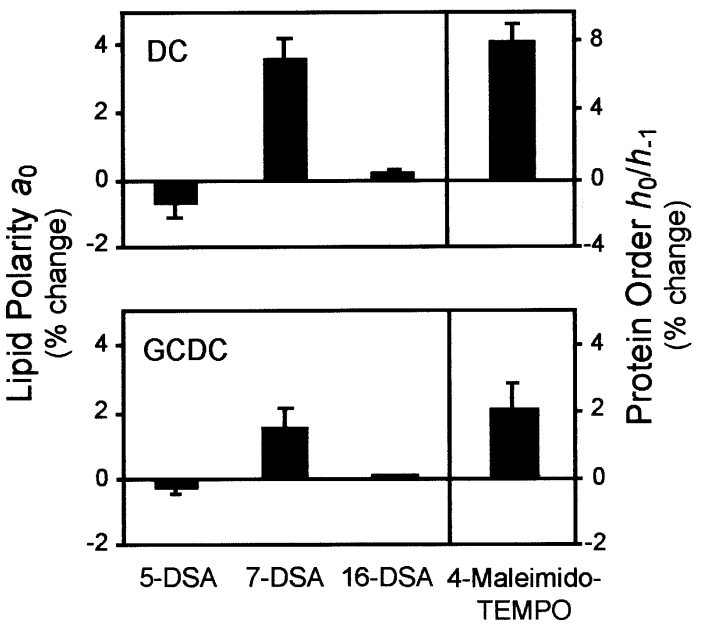

Figure I Hydrophobic bile salts influence lipid polarity and protein order, at the surface level, in rat liver mitochondrial membranes

Mitochondria were labelled with 5-DSA, 7-DSA, 16-DSA or 4-maleimido-TEMPO spin probes and exposed to $200 \mu \mathrm{M} \mathrm{DC}, 200 \mu \mathrm{M} \mathrm{GCDC}$ or vehicle only (control) for $5 \mathrm{~min}$ as described in the Materials and methods section. Mitochondrial pellets were examined for lipid polarity $\left(a_{0}\right)$ and protein order $\left(h_{0} / h_{-1}\right)$ by EPR spectroscopy analyses. Values are means \pm S.E.M. from at least four separate experiments of the percentage change relative to controls.

cumulative and dependent on concentration and exposure time. Thus higher concentrations are necessary to evaluate toxicity after the short incubation periods required due to the instability of isolated mitochondria. We have chosen to use a $200 \mu \mathrm{M}$ concentration of hydrophobic bile salts for most experiments, because similar concentrations specifically impair mitochondrial respiration and increase membrane permeability in isolated organelles [7,12,14,15]. In addition, lower bile salt levels did not significantly change EPR spectra after a 5 min exposure (results not shown).

Labelled mitochondria were treated with $200 \mu \mathrm{M} \mathrm{DC}$ or GCDC for $5 \mathrm{~min}$, and then assayed for lipid polarity at various depths (Figure 1). As sensed superficially by the 5-DSA probe, DC induced a significant decline in membrane polarity $(P<0.05)$, while GCDC treatment resulted in only marginal changes. In contrast, very marked increases in lipid polarity were observed in mitochondria labelled with the 7-DSA probe and then exposed to DC $(P<0.01)$ or GCDC $(P<0.05)$. A slight perturbation was also indicated by the 16-DSA spin label. Because the latter probe is localized deeper in the membrane leaflet, these data suggest that more hydrophobic membrane regions are barely affected after short incubations of isolated liver mitochondria with lower DC or GCDC concentrations. Further, the surface membrane perturbation was greater with bile salt concentrations of $>200 \mu \mathrm{M}$, but was always more evident for DC, while deeper structural disruption only attained 
Table I Disruption of lipid fluidity at various depths in isolated rat liver mitochondria exposed to hydrophobic bile salts

Spin-labelled mitochondria were exposed to $200 \mu \mathrm{M} \mathrm{DC}, 200 \mu \mathrm{M} \mathrm{GCDC}$ or vehicle only (control), for $5 \mathrm{~min}$, in respiration buffer as described in the Materials and methods section. Alterations in outer half-width at half-height of the low-field extreme $(\Delta /)$ were sensed by the 5-DSA and 7-DSA spin labels, while alterations in the ratio of the heights of the low-field and the centre-field lines $\left(h_{+1} / h_{0}\right)$ were sensed by the 16-DSA spin label. Percentage decreases in lipid fluidity at $C-5$ and percentage increases at $\mathrm{C}-7$ and $\mathrm{C}-16$ were calculated based on the corresponding control values. Values are means \pm S.E.M. of at least four separate experiments.

\begin{tabular}{llll}
\hline & \multicolumn{2}{l}{ Change in lipid fluidity (\%) } \\
\cline { 2 - 3 } & \multicolumn{4}{c}{} & \\
\cline { 2 - 4 } & 5 -DSA & 7 -DSA & 16-DSA \\
\hline Bile salt & $-4.2 \pm 1.7$ & $8.2 \pm 1.5$ & $2.2 \pm 0.2$ \\
\hline DC & $-2.6 \pm 1.8$ & $5.1 \pm 3.3$ & $2.0 \pm 0.4$ \\
GCDC & & & \\
\hline
\end{tabular}

statistical significance at these concentrations (results not shown).

Hydrophobic bile salts at the lowest concentration also increased membrane lipid order in the local microenvironment reported by the 5-DSA spin label. In contrast, using the 7-DSA spin label, DC and GCDC caused a marked elevation in $\Delta l$, indicative of increased membrane motion or fluidity, while deeper in the membrane leaflet, $h_{+1} / h_{0}$ was only slightly raised, as revealed by the 16-DSA probe. Table 1 shows the values of the motion parameters $\Delta l$ and $h_{+1} / h_{0}$ expressed as a percentage of control to identify the membrane region most affected. In fact, lipid fluidity was slightly decreased at C-5 $(P<0.05)$, but markedly enhanced at C-7 $(P<$ 0.01 ), thus corroborating the information given by the parameter $a_{0}$.

In parallel experiments, the 4-maleimido-TEMPO spin label was used to gain insight into the protein order structure of mitochondria (Figure 1). After a $5 \mathrm{~min}$ exposure to lower concentrations of DC or GCDC, the order of superficial proteins was increased and significantly different from untreated controls $(P<0.01$ and $P<0.05$ respectively). These data are consistent with decreased protein mobility, and provide independent evidence for the proposed interaction of hydrophobic bile salts with the superficial domain of mitochondrial membranes sensed by lipid-oriented spin labels. Further, concentrations of hydrophobic bile salts greater than $200 \mu \mathrm{M}$ markedly decreased protein order $(P<0.01)$, probably reflecting the solubilization of membrane components.

To determine whether changes in membrane lipid polarity and fluidity could be reversed by hydrophilic bile salts, we analysed membrane perturbations sensed by the 7-DSA spin label using DC in the presence or absence
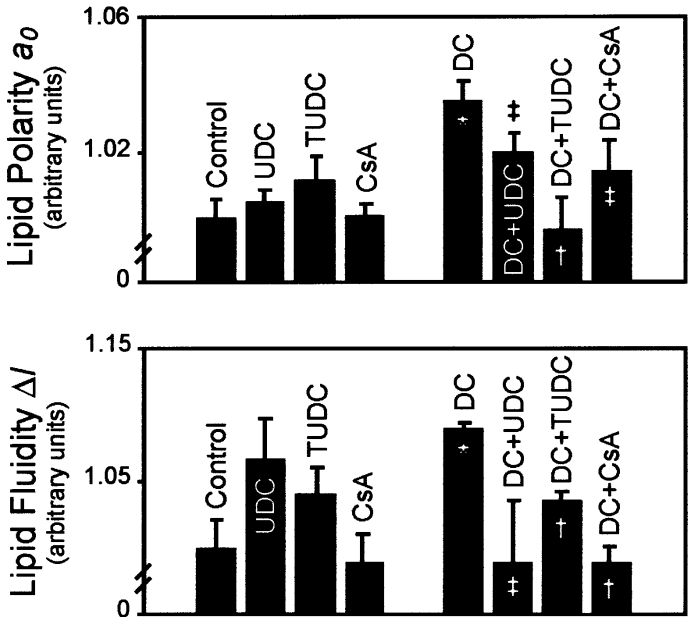

Figure 2 Hydrophilic bile salts and cyclosporin A abrogate disruption of lipid polarity and fluidity induced by $D C$ in rat liver mitochondrial membranes

Labelled mitochondria were exposed to $200 \mu \mathrm{M} \mathrm{DC}, 500 \mu \mathrm{M}$ UDC, $500 \mu \mathrm{M}$ TUDC, $5 \mu \mathrm{M}$ cyclosporin $\mathrm{A}(\mathrm{CSA}), \mathrm{DC}$ plus UDC, DC plus TUDC, DC plus CsA or vehicle only (control) for $5 \mathrm{~min}$ as described in the Materials and methods section. In coincubation experiments, mitochondria were pretreated with UDC, TUDC or CSA for $5 \mathrm{~min}$. Mitochondrial pellets were examined for lipid polarity $\left(a_{0}\right)$ and fluidity $(\Delta /)$ sensed by the 7-DSA spin label using EPR spectroscopy analysis. Values are means \pm S.E.M. from at least four separate experiments, relative to controls. Significance of differences: $\ddagger P<0.05, \uparrow P<0.01$ compared with $D C$ alone; ${ }^{*} P<0.01$ compared with control.

of UDC or TUDC (Figure 2). The dynamic properties of mitochondrial membranes at this level were only slightly altered by UDC or TUDC alone compared with untreated organelles. However, marked increases in membrane permeability and motion induced by DC $(P<0.01)$ were significantly prevented by the hydrophilic species, with levels of protection ranging from $40 \%$ to almost $100 \%$. Similarly, cyclosporin A, an inhibitor of megapore opening, inhibited DC-induced membrane permeabilization by at least $60 \%(P<0.05)$. Finally, using the 4 maleimido-TEMPO label, UDC alone decreased protein mobility by $>10 \%(P<0.001)$, suggesting a direct effect of the hydrophilic bile salt on the organization of surface membrane proteins. Co-incubation with hydrophilic and hydrophobic bile salts also augmented protein order and, therefore, decreased protein mobility $(P<0.05)$.

\section{Is perturbation of mitochondrial membrane structure by hydrophobic bile salts associated with cytochrome $c$ release?}

Because hydrophobic bile salts effectively disrupted superficial lipid and protein membrane structure in isolated mitochondria, we sought to determine whether this perturbation was directly associated with the release of intermembrane proteins. We addressed this question by measuring levels of cytochrome $c$, using immunoblot analysis, in mitochondrial pellets and supernatants after 


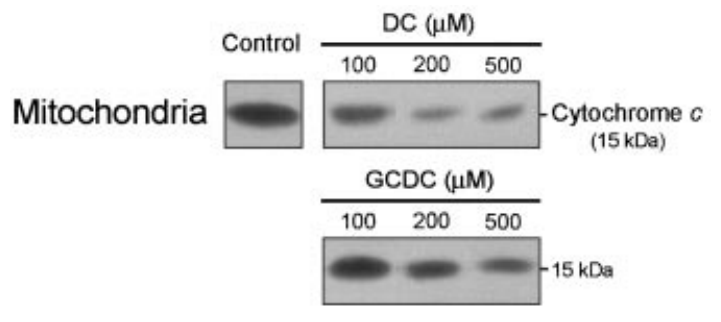

Figure 3 DC and GCDC induce a concentration-dependent release of cytochrome $c$ from isolated rat liver mitochondria Organelles were exposed to 100,200 or $500 \mu \mathrm{MDC}$ or $\mathrm{GCDC}$, or to vehicle only (control), for $5 \mathrm{~min}$ as described in the Materials and methods section. Mitochondrial pellets were examined for cytochrome $c$ levels by Western blot analysis. Following SDS/PAGE and transfer, nitrocellulose membranes were incubated with a monoclonal antibody to cytochrome $c$, and the $15 \mathrm{kDa}$ protein was detected using $\mathrm{ECL}^{\circledR}$.

incubation with various concentrations of DC or GCDC. Cytochrome $c$ was abundant in control mitochondria, but levels decreased progressively after exposure to 100, 200 and $500 \mu \mathrm{M} \mathrm{DC}$ or GCDC (Figure 3), corresponding to a proportional increase in supernatant levels $(P<$ 0.01). Thus cytochrome $c$ release at concentrations of hydrophobic bile salts slightly lower than those necessary to induce structural changes suggest that the efflux of intermembrane proteins is a primary event that may further disrupt mitochondrial membrane structure, again increasing permeability, in a vicious cycle. Further, these data support a role for a specific injury rather than nonspecific effects of these detergents, which was also confirmed by a marked cyclosporin A-sensitive decrease in cytochrome $c$ levels in mitochondria suspensions treated with hydrophobic bile salts $(P<0.01)$. Finally, as shown in previous studies, cytochrome $c$ efflux was reduced by $>70 \%$ during pretreatment with either UDC or TUDC $(P<0.01)$.

Of note is the observation that the release of glutamate dehydrogenase from the mitochondrial matrix after a 5 min exposure to DC and GCDC, at lower concentrations, induced only $5 \%$ and $1 \%$ increases respectively in enzyme release. In addition, these bile salts directly caused mitochondrial release of cytochrome $c$, but not cytochrome $c$ oxidase (results not shown), corroborating the hypothesis that the cytotoxicity of these bile salts may be associated with specific physical perturbation of the mitochondrial membrane, and not simply organelle bursting.

\section{Is the increase in permeability of mitochondrial membranes induced by hydrophobic bile salts associated with altered redox status?}

To determine if exposure to DC or GCDC modifies the levels of mitochondrial membrane oxidation, isolated
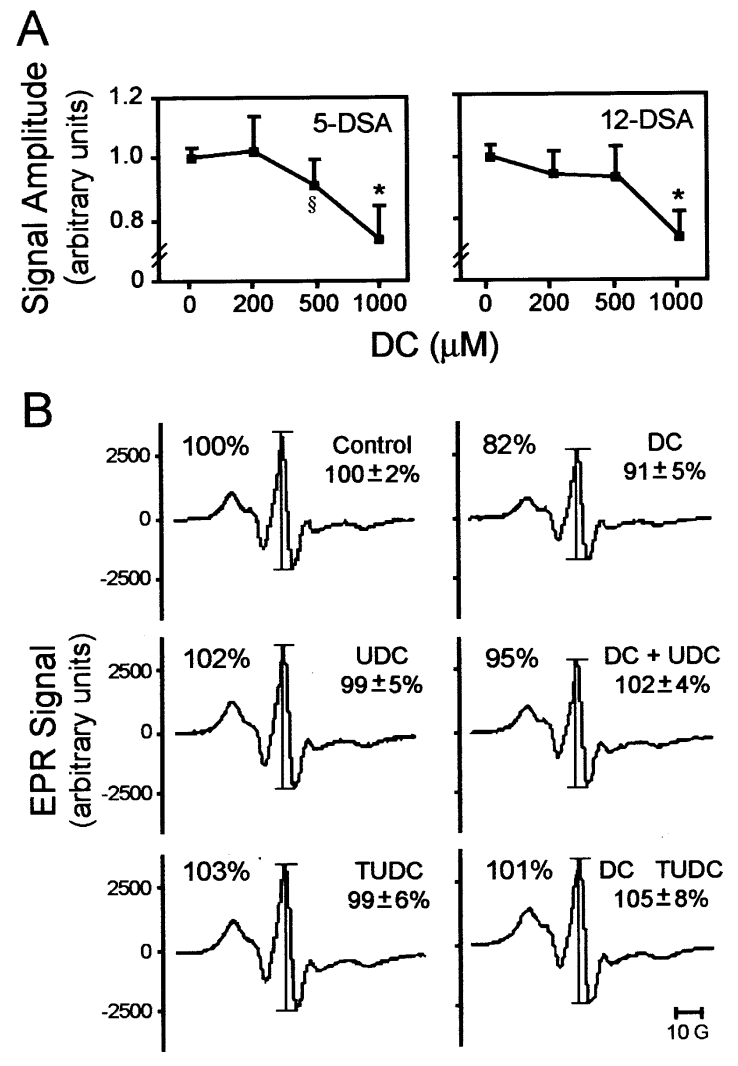

Figure 4 DC induces oxidative damage of lipids in rat liver mitochondrial membranes

Mitochondria were labelled with either 5-DSA or I2-DSA spin probes and exposed to $D C$ for $5 \mathrm{~min}$ as described in the Materials and methods section. Mitochondrial pellets were examined for free radical production using EPR spectroscopy analysis. (A) Dose-response to DC-induced decrease in signal amplitude sensed by 5 - and I2-DSA spin labels. Labelled mitochondria were exposed to either DC (200, 500 or $1000 \mu \mathrm{M})$ or vehicle only $(0 \mu \mathrm{M}$; control). (B) Alteration in EPR spectra sensed by the 5-DSA spin label. Labelled mitochondria were exposed to $500 \mu \mathrm{M} \mathrm{DC}$, $500 \mu \mathrm{M} U \mathrm{DCC}, 500 \mu \mathrm{M}$ TUDC, DC plus UDC, DC plus TUDC or vehicle only (control). In co-incubation experiments, mitochondria were pretreated with either UDC or TUDC for $5 \mathrm{~min}$. Values are means \pm S.E.M. from at least four separate experiments relative to controls. Significance of differences: $\$ P<0.05,{ }^{*} P<0.01$ compared with control.

mitochondria were probed with 5-DSA and 12-DSA spin labels. The results showed that DC induced a sudden, dose-dependent loss of spin-probe intensity, manifested by a decrease in peak amplitude, which is promoted by the reaction between the toxicity-associated free radicals and the unpaired electron on the nitroxide spin probes. Exposure to $200 \mu \mathrm{M} \mathrm{DC}$ for $5 \mathrm{~min}$ did not significantly alter 5-DSA signal amplitude, while $500 \mu \mathrm{M}$ caused a marked decrease in spin-probe intensity $(P<0.05)$ (Figure $4 \mathrm{~A}$ ), indicating that oxidative stress is most likely to be a consequence of increased mitochondrial membrane permeability. The magnitude of the 12-DSA signal incorporated into mitochondrial membranes was also decreased during exposure to DC, although to a lesser 
extent. These results indicate less oxidative damage in deeper regions of the bilayer, and support a specific superficial effect. Finally, perturbation induced by GCDC was rapid, but only significant at higher concentrations (results not shown).

Spin-label signal amplitude in isolated mitochondria was also assessed in the presence of UDC or TUDC (Figure 4B). Treatment of 5-DSA-labelled mitochondria with each hydrophilic bile salt alone did not significantly decrease signal amplitude, while incubation with UDC or TUDC for $5 \mathrm{~min}$ prior to exposure to DC completely abrogated oxidative injury $(P<0.01)$. This protection resulted in signal amplitudes that were no different from those of controls, indicating that the hydrophilic bile salts either confer direct protection against lipid peroxidation in isolated mitochondrial membranes or prevent the insertion of hydrophobic bile salts into membranes, indirectly inhibiting free radical production.

\section{DISCUSSION}

The mechanisms of hepatocellular damage during liver disease are not entirely understood, but appear to be related to the retention of toxic substances. It is widely recognized that accumulation of hydrophobic bile salts within the hepatocyte plays a key part in liver injury [1-3], where the prominence of hepatocyte-derived acidophilic bodies and cell dropout, rather than extensive necrosis, is supportive of a role for apoptosis $[4,5,23]$. In addition, exposure of cells to toxic bile salts has been shown to cause apoptosis, both in vivo and in vitro, in a concentration- and time-dependent manner, at concentrations that are far lower than the critical micellar concentration associated with cell necrosis $[6,7]$. Finally, several studies have shown that bile salt-induced cytotoxicity may be a consequence of mitochondrial dysfunction. Short-time incubations of isolated rat liver mitochondria with hydrophobic bile salts causes impaired mitochondrial respiration [12] as well as morphological changes associated with the opening of the mitochondrial permeability transition pore [7,13-15], suggesting a specific membrane perturbation. The nature of membrane changes and the sequence of events that occur in mitochondria remain, however, poorly defined, in part because of the intrinsic difficulty [39] of measuring the dynamic properties of mitochondrial membranes.

The detailed mechanism(s) by which hydrophobic bile salts increase membrane permeability in isolated mitochondria is not currently understood. Cyclosporin A, which is commonly used to prevent the mitochondrial permeability transition, has been reported to inhibit cytochrome $c$ release from mitochondria treated with hydrophobic bile salts [7,13-15]. This observation raises the possibility that DC and GCDC may interact with membrane components, promoting the release of inter- membrane proteins through a cyclosporin A-sensitive megapore. The present study demonstrates a direct physical interaction between hydrophobic bile salts and mitochondrial membranes. Indeed, membrane lipid polarity and fluidity were particularly disturbed near the lipid-water interface, more than in hydrophobic regions, suggesting a specific effect rather than non-specific changes. The decreased mitochondrial membrane polarity and fluidity reported by the 5-DSA spin label suggests that hydrophobic bile salts intercalated at the C5 membrane region, increasing phospholipid packing and thus reducing the already low mobility of the phospholipid acyl chain in this region of the leaflet. This was confirmed by the order parameter $S$. The superficial effect described above would thus render inner regions of the membrane more permeable, as indicated by the increased $a_{0}$ value sensed by either 7-DSA or, to a much lower extent, 16-DSA. Further, since perturbation of the mitochondrial membrane induced by hydrophobic bile salts decreased with increasing depth of the leaflet from C-7 to C-16, it is conceivable that this gradient may result from an effect secondary to the superficial accommodation of toxic bile salts. This scenario is not without precedent, since similar effects have been described for other membrane-disturbing agents $[40,41]$. Finally, exposure to bile salt concentrations greater than $200 \mu \mathrm{M}$ resulted in increased perturbation sensed by all spin labels; this was, however, proportionally greater in deeper regions, probably reflecting lipid solubilization due to detergent effects. To this end, studies using erythrocyte and hepatocyte membranes also showed marked spectral alterations in apolar regions induced by a different hydrophobic bile salt, unconjugated chenodeoxycholate, at concentrations ranging from 250 to $1000 \mu \mathrm{M}$ [42]. Moreover, the increased membrane polarity after incubation with higher bile salt concentrations has been described to correlate with enhanced solubilization of cholesterol and phospholipids [42]. Interestingly, the superficial interaction of hydrophobic bile salts sensed by lipid spin labels may explain the observation that DC and GCDC decreased the mobility of the 4-maleimidoTEMPO label, thus reflecting augmented superficial protein order structure above control values. As expected, higher bile salt concentrations led to increased mobility of this spin label and, therefore, to enhanced membrane fluidity, in accordance with suggestions by other authors [28].

Further experiments were performed to determine how toxic bile salts might be affecting mitochondrial function. We observed that changes in mitochondrial membrane structure were evident after cytochrome $c$ release, resulting in subsequent oxidative lipid damage. This sequence of events is corroborated by the presence of structural changes at bile salt concentrations higher than those necessary to induce cytochrome $c$ release, and by the occurrence of oxidative damage at even greater 
concentrations. It appears that the release of cytochrome $c$ may further disrupt membrane structure, again increasing permeability, in a vicious cycle. Moreover, oxidative damage seems to be a consequence of cytochrome $c$ release rather than a promoter of it, whereby the absence of cytochrome $c$ may inhibit electron transfer, disrupting the respiratory chain.

The paramagnetic signal of 5-DSA was markedly lowered in DC-treated mitochondria in particular, reflecting free radical production, while at C-12 the same effect was slightly attenuated. These findings are supported by other studies showing that hydrophobic bile salts promote oxidative stress $[7,8,43,44]$, and are also consistent with signs of diminished injury during treatment with antioxidants $[45,46]$.

To determine whether hydrophilic bile salts may play a role in preventing DC- and GCDC-triggered membrane perturbation, isolated mitochondria were pretreated with UDC or TUDC. These hydrophilic bile salts almost completely reversed both the disruption of mitochondrial membrane structure and the oxidative damage caused by the hydrophobic species. The protective role of UDC and TUDC at both the C-5 and C7 levels of the mitochondrial membrane also suggests interaction at superficial membrane domains. However, this explanation does not exclude the possibility that UDC might directly influence protein function, as suggested by the marked effect on protein order reported by the 4-maleimido-TEMPO spin label, or might also interact with deeper regions of the membrane, as reported by others [28]. Most probably, the binding sites of the various bile salts are not the same. As previously suggested, the stabilization of the mitochondrial membrane by UDC and TUDC implied here provides an additional explanation for the protective role of these bile salts in preventing apoptosis in several model systems $[7,15,47]$. Similar observations were made when mitochondria were preincubated with cyclosporin A. This inhibitor of the megapore opening prevents cytochrome $c$ release induced by several toxic agents $[48,49]$, precluding the involvement of the mitochondrial permeability transition during induction of cytotoxicity by hydrophobic bile salts.

Collectively, data from the present study appear to suggest a mechanism of toxicity that involves rapid perturbation of superficial mitochondrial membrane structure during incubation with hydrophobic bile salts, resulting in increased membrane permeability and oxidative injury. UDC, TUDC and cyclosporin A almost completely abrogated DC- and GCDC-induced perturbation of mitochondrial membrane structure. These observations imply that the cytotoxicity of hydrophobic bile salts may be mediated, at least in part, by their effect of physically disturbing the mitochondrial membrane rather than by an influence on the expression of proapoptotic Bax. Perturbation of mitochondrial membrane structure may indeed represent an important component of the apoptotic pathway triggered by accumulated bile salts during liver disease, confirming mitochondria as primary targets of bile salt-induced cytotoxicity. Thus new prospects for the treatment of liver diseases should consider the use of molecules that, like UDC and its conjugated derivatives, stabilize the mitochondrial membrane.

\section{ACKNOWLEDGMENTS}

We thank Carlos D. Brondino (Departamento de Química, FCT, Universidade Nova de Lisboa, Monte de Caparica, Portugal) for his expertise in the use of the EPR spectrometer. This work was supported in part by grant PRAXIS/C/SAU/14311/1998 from Fundação para a Ciência e a Tecnologia, Lisbon, Portugal, and by a Research Fellowship 2000 from the European Association for the Study of the Liver (to C.M.P.R.).

\section{REFERENCES}

1 Scholmerich, J., Becher, M. S., Schmidt, K. et al. (1984) Influence of hydroxylation and conjugation of bile salts on their membrane-damaging properties - studies on isolated hepatocytes and lipid membrane vesicles. Hepatology 4, 661-666

2 Schmucker, D. L., Ohta, M., Kanai, S., Sato, Y. and Kitani, K. (1990) Hepatic injury induced by bile salts: correlation between biochemical and morphological events. Hepatology 12, 1216-1221

3 Heuman, D. M., Mills, A. S., Mccall, J., Hylemon, P. B., Pandak, W. M. and Vlahcevic, Z. R. (1991) Conjugates of ursodeoxycholate protect against cholestasis and hepatocellular necrosis caused by more hydrophobic bile salts. In vivo studies in the rat. Gastroenterology 100, 203-211

4 Columbano, A. (1995) Cell death: current difficulties in discriminating apoptosis from necrosis in the context of pathological processes in vivo. J. Cell. Biochem. 58, $181-190$

5 Patel, T. and Gores, G. J. (1995) Apoptosis and hepatobiliary disease. Hepatology 21, 1725-1741

6 Patel, T., Bronk, S. F. and Gores, G. J. (1994) Increases of intracellular magnesium promote glycodeoxycholateinduced apoptosis in rat hepatocytes. J. Clin. Invest. 94, 2183-2192

7 Rodrigues, C. M. P., Fan, G., Ma, X., Kren, B. T. and Steer, C. J. (1998) A novel role for ursodeoxycholic acid in inhibiting apoptosis by modulating mitochondrial membrane perturbation. J. Clin. Invest. 101, 2790-2799

8 Yerushalmi, B., Dahl, R., Devereaux, M. W., Gumpricht, E. and Sokol, R. J. (2001) Bile acid-induced rat hepatocyte apoptosis is inhibited by antioxidants and blockers of the mitochondrial permeability transition. Hepatology 33, 616-626

9 Schaffner, F., Bacchin, P. G., Hutterer, F. et al. (1971) Mechanism of cholestasis. 4. Structural and biochemical changes in the liver and serum in rats after bile duct ligation. Gastroenterology 60, 888-897

10 Krähenbühl, S., Stucki, J. and Reichen, J. (1992) Reduced activity of the electron transport chain in liver mitochondria isolated from rats with secondary biliary cirrhosis. Hepatology 15, 1160-1166

11 Spivey, J. R., Bronk, S. F. and Gores, G. J. (1993) Glycochenodeoxycholate-induced lethal hepatocellular injury in rat hepatocytes. Role of ATP depletion and cytosolic free calcium. J. Clin. Invest. 92, 17-24 
12 Krähenbühl, S., Talos, C., Fischer, S. and Reichen, J. (1994) Toxicity of bile acids on the electron transport chain of isolated rat liver mitochondria. Hepatology 19, 471-479

13 Botla, R., Spivey, J. R., Aguilar, H., Bronk, S. F. and Gores, G. J. (1995) Ursodeoxycholate (UDCA) inhibits the mitochondrial membrane permeability transition induced by glycochenodeoxycholate: a mechanism of UDCA cytoprotection. J. Pharmacol. Exp. Ther. 272, 930-938

14 Rodrigues, C. M. P., Fan, G., Wong, P. Y., Kren, B. T. and Steer, C. J. (1998) Ursodeoxycholic acid may inhibit deoxycholic acid-induced apoptosis by modulating mitochondrial transmembrane potential and reactive oxygen species production. Mol. Med. 4, 165-178

15 Rodrigues, C. M. P., Ma, X., Linehan-Stieers, C., Fan, G. Kren, B. T. and Steer, C. J. (1999) Ursodeoxycholic acid prevents cytochrome c release in apoptosis by inhibiting mitochondrial membrane depolarization and channel formation. Cell Death Differ. 6, 842-854

16 Lieser, M. J., Park, J., Natori, S., Jones, B. A., Bronk, S. F. and Gores, G. J. (1998) Cholestasis confers resistance to the rat liver mitochondrial permeability transition. Gastroenterology 115, 693-701

17 Degterev, A., Boyce, M. and Yuan, J. (2001) The channel of death. J. Cell Biol. 155, 695-698

18 De Giorgi, F., Lartigue, L., Bauer, M. K. A. et al. (2002) The permeability transition pore signals apoptosis by directing Bax translocation and multimerization. FASEB J. 16, 607-609

19 Liu, X., Kim, C. N., Yang, J., Jemmerson, R. and Wang, $X$. (1996) Induction of apoptotic program in cell-free extracts: requirement for dATP and cytochrome c. Cell 86, 147-157

20 Zou, H., Henzel, W. J., Liu, X., Lutschg, A. and Wang, X. (1997) Apaf-1, a human protein homologous to C. Elegans Ced-4, participates in cytochrome c-dependent activation of caspase-3. Cell $90,405-413$

21 Kroemer, G. (1997) The proto-oncogene bcl-2 and its role in regulating apoptosis. Nat. Med. (N.Y.) 3, 614-620

22 Beuers, U., Boyer, J. L. and Paumgartner, G. (1998) Ursodeoxycholic acid in cholestasis: potential mechanisms of action and therapeutic applications. Hepatology 28, 1449-1453

23 Rodrigues, C. M. and Steer, C. J. (2000) Mitochondrial membrane perturbations in cholestasis. J. Hepatol. 32, 135-141

24 Azzaroli, F., Mehal, W., Soroka, C. J. et al. (2002) Ursodeoxycholic acid diminishes Fas-ligand-induced apoptosis in mouse hepatocytes. Hepatology 36, 49-54

25 Mariash, C. N., Seelig, S., Schwartz, H. L. and Oppenheimer, J. H. (1986) Rapid synergistic interaction between thyroid hormone and carbohydrate on mRNAS14 induction. J. Biol. Chem. 261, 9583-9586

26 Jost, P., Libertini, L. J., Hebert, V. C. and Griffith, O. H. (1971) Lipid spin labels in lecithin multilayers. A study of motion along fatty acid chains. J. Mol. Biol. 59, 77-98

27 Harris, J., Power, T. J., Bieber, A. L. and Watts, A. (1983) An electron-spin-resonance spin-label study of the interaction of purified mojave toxin with synaptosomal membranes from rat brain. Eur. J. Biochem. 131, 559-565

28 Güldutüna, S., Zimmer, G., Leuschner, M. et al. (1999) The effect of bile salts and calcium on isolated rat liver mitochondria. Biochim. Biophys. Acta 1453, 396-406

29 Mason, R. P., Giavedoni, E. B. and Dalmasso, A. P. (1977) Complement-induced decrease in membrane mobility: introducing a more sensitive index of spin-label motion. Biochemistry 16, 1196-1201

30 Hubbell, W. L. and Mcconnell, M. (1971) Molecular motion in spin-labeled phospholipids and membranes. J. Am. Chem. Soc. 93, 314-326

31 Schreier, S., Frezzatti, W. A., Araujo, P. S., Chaimovich, H. and Cuccovia, I. M. (1984) Effect of lipid membranes on the apparent pk of the local anesthetic tetracaine. Spin label and titration studies. Biochim. Biophys. Acta 769, 231-237
32 Bianconi, M. L., do Amaral, A. T. and Schreier, S. (1988) Use of membrane spin label spectra to monitor rates of reaction of partitioning compounds: hydrolysis of a local anesthetic analog. Biochem. Biophys. Res. Commun. 152, 344-350

33 Lai, C. S., Tooney, N. M. and Ankel, E. G. (1984) Structure and flexibility of plasma fibronectin in solution: electron spin resonance spin-label, circular dichroism, and sedimentation studies. Biochemistry 23, 6393-6397

34 Kunicki, T. J., Nugent, D. J., Piotrowicz, R. S. and Lai, C. S. (1986) Covalent attachment of sulfhydryl-specific, electron spin resonance spin-labels to fab' fragments of murine monoclonal antibodies that recognize human platelet membrane glycoproteins. Development of membrane protein specific spin probes. Biochemistry 25, 4979-4983

35 Bruce-Keller, A. J., Begley, J. G., Fu, W. et al. (1998) Bcl2 protects isolated plasma and mitochondrial membranes against lipid peroxidation induced by hydrogen peroxide and amyloid beta-peptide. J. Neurochem. 70, 31-39

36 Butterfield, D. A., Hensley, K., Harris, M., Mattson, M. and Carney, J. (1994) $\beta$-amyloid peptide free radical fragments initiate synaptosomal lipoperoxidation in a sequence-specific fashion: implications to Alzheimer's disease. Biochem. Biophys. Res. Commun. 200, 710-715

37 Hensley, K., Carney, J., Hall, N., Shaw, W. and Butterfield, D. A. (1994) Electron paramagnetic resonance investigations of free radical-induced alterations in neocortical synaptosomal membrane protein infrastructure. Free Radical Biol. Med. 17, 321-331

38 Roda, A., Minutello, A., Angellotti, M. A. and Fini, A. (1990) Bile acid structure-activity relationship: evaluation of bile acid lipophilicity using 1-octanol/water partition coefficient and reverse phase HPLC. J. Lipid Res. 31, 1433-1443

39 Bernardi, P., Scorrano, L., Colonna, R., Petronilli, V. and Di Lisa, F. (1999) Mitochondria and cell death. Mechanistic aspects and methodological issues. Eur. J. Biochem. 264, 687-701

40 Ondrias, K., Staško, A. and Balgavý, P. (1987) Spin label study of the perturbation effect of the local anaesthetics tetracaine and dibucaine on synaptosomes at pharmacological concentrations. Biochem. Pharmacol. 36, 3999-4005

41 Brito, M. A., Brondino, C. D., Moura, J. J. G. and Brites, D. (2001) Effects of bilirubin molecular species on membrane dynamic properties of human erythrocyte membranes: a spin label electron paramagnetic resonance spectroscopy study. Arch. Biochem. Biophys. 387, 57-65

42 Güldutüna, S., Zimmer, G., Imhof, M., Bhatti, S., You, T. and Leuschner, U. (1993) Molecular aspects of membrane stabilization by ursodeoxycholate. Gastroenterology 104, 1736-1744

43 Sokol, R. J., Devereaux, M. W., Khandwala, R. and O'Brien, K. (1993) Evidence for involvement of oxygen free radicals in bile acid toxicity to isolated rat hepatocytes. Hepatology 17, 869-881

44 Sokol, R. J., Winklhofer-Roob, B. M., Devereaux, M. W. and McKim, Jr, J. M. (1995) Generation of hydroperoxides in isolated rat hepatocytes and hepatic mitochondria exposed to hydrophobic bile acids. Gastroenterology 109, 1249-1256

45 Sokol, R. J., Devereaux, M. W. and Khandwala, R. (1991) Effect of dietary lipid and vitamin $\mathrm{E}$ on mitochondrial lipid peroxidation and hepatic injury in the bile ductligated rat. J. Lipid Res. 32, 1349-1357

46 Sokol, R. J., McKim, J. M., Goff, M. C. et al. (1998) Vitamin E reduces oxidant injury to mitochondria and the hepatotoxicity of taurochenodeoxycholic acid in the rat. Gastroenterology 114, 164-174

47 Rodrigues, C. M. P., Stieers, C. L., Keene, C. D. et al. (2000) Tauroursodeoxycholic acid partially prevents apoptosis induced by 3-nitropropionic acid: evidence for a mitochondrial pathway independent of the permeability transition. J. Neurochem. 75, 2368-2379 
48 Pastorino, J. G., Synder, J. W., Serroni, A., Hoek, J. B. and Farber, J. L. (1993) Cyclosporine and carnitine prevent the anoxic death of cultured hepatocytes by inhibiting the mitochondrial permeability transition. J. Biol. Chem. 268, 13791-13798
49 Bernardi, P. (1992) Modulation of the mitochondrial cyclosporine A-sensitive permeability transition pore by the proton electrochemical gradient. Evidence that the pore can be opened by membrane depolarization. J. Biol. Chem. 267, 8834-8839

Received 17 July 2002; accepted 8 August 2002 